\title{
BMJ Open Retrospective cohort study of compliance with post-deployment screening in the Canadian Armed Forces
}

\author{
Peter J H Beliveau, David Boulos, Dylan Johnson
}

To cite: Beliveau PJH, Boulos D, Johnson D. Retrospective cohort study of compliance with post-deployment screening in the Canadian Armed Forces. BMJ Open 2019;9:e029355. doi:10.1136/ bmjopen-2019-029355

- Prepublication history for this paper is available online. To view these files, please visit the journal online (http://dx.doi org/10.1136/bmjopen-2019029355).

Received 22 January 2019 Revised 4 April 2019 Accepted 5 June 2019

\section{Check for updates}

(c) Author(s) (or their employer(s)) 2019. Re-use permitted under CC BY-NC. No commercial re-use. See rights and permissions. Published by BMJ.

Directorate of Mental Health, Canadian Forces Health Services Group, Ottawa, Ontario, Canada

Correspondence to

David Boulos;

david.boulos@forces.gc.ca

\section{ABSTRACT}

Objective Canadian Armed Forces (CAF) personnel who return from certain international deployments are required to complete post-deployment screening (PDS) 90 to 180 days post-deployment; the primary goal of PDS is early detection of mental health problems that aims for reduced delays to care provision. We investigated service members' compliance with the PDS completion requirement and the factors associated with this compliance; a secondary objective was to investigate completion timing. Design The study used a retrospective cohort of CAF personnel $(n=28460)$ who had deployments over 01 January 2009 to 31 December 2014; inferences were based on a probabilistic sample $(n=3004)$.

Primary outcome The primary outcome was PDS completion. We assessed the timing of PDS completion, comparing non-compliant (early, late or no completion) with compliant completions (90 to 180 days postdeployment) among deployments that required screening. Kaplan-Meier plots summarised time-to-completion and logistic regression assessed the covariate associations with compliant completion. Covariate-adjusted marginal compliance prevalence differences (MPD) were computed. Results $67.3 \%$ (95\% Cl65.0 to 69.6) of deployments that required PDS had one completed; $43.3 \%(95 \% \mathrm{Cl}$ 40.6 to 46.0) were completed within the compliant period. Compliant completion was higher with lower ranks (MPD $=10.6 \%$, relative to officers), combat arms occupations (MPD=8.4\%), Afghanistan deployments (MPD $=19.2 \%)$, longer deployments $(\mathrm{MPD}=10.1 \%)$ and among those without a past mental health problem (MPD=9.7\%).

Conclusions Our findings suggest that some selective processes may be occurring where those perceived to be at higher risk for post-deployment mental health problems are more compliant with PDS completion. However, PDS completion and compliant completion were lower than expected and this suggests a need to reinforce instruction on the guidelines and objectives of PDS among service members in the CAF.

\section{INTRODUCTION}

Military personnel encounter unique experiences during their service and these can result in an increased vulnerability to developing mental health problems; this is especially evident among those returning from difficult deployments. ${ }^{1-4}$ In response to this awareness,

\section{Strengths and limitations of this study}

The primary limitation of our study relates to it being retrospective and as such, we were reliant on the information that was available.

- We were unable to identify specific individual reasons for non-compliance with screening.

- An unknown number of individuals may have started the screening process prior to the required 180 day post-deployment period but finished it after this time point, or started without continuing on to complete the interview; these individuals would have been classified as either non-compliant late completers or non-completers.

- While screening in the Canadian Armed Forces (CAF) requires that personnel returning from requisite deployments complete post-deployment screening 90 to 180 days after deployment return, this study and assessment was not designed to determine whether this screening window is optimal with respect to desired outcomes for the CAF setting.

a number of countries - including the USA and Canada - have reinforced their military mental health systems and implemented interventions to assist their personnel. Some militaries have implemented post-deployment screening (PDS) programmes in an attempt to reduce the suffering and expedite care provision for service members who may be experiencing mental health concerns. ${ }^{4-9}$

There are a number of potential benefits to PDS and these are largely associated with a required interaction with mental health services soon after return. Mental health problems among military personnel can be impactful to the individual and the organisation as it can preclude future deployments, reduce operational readiness and lead to a medical release from service. ${ }^{10}$ Studies on the association of mental disorder prognosis relative to the timing of care are sparse but some findings suggest that earlier treatment after deployment return is associated with a greater likelihood of symptom improvement ${ }^{11}$ and a more favourable occupational outcome. ${ }^{12}$ 
Additionally, there is potentially a cost benefit associated with earlier treatment; for example, annual health services costs can be significantly lower for patients using early intervention services compared with non-users. ${ }^{13}$ Moreover, one of the leading barriers to accessing mental healthcare in military populations is a failure to perceive a need for care and this can be offset with screening. ${ }^{14}$ This is especially true when a large fraction of military personnel with a probable mental disorder do not seek help ${ }^{15-17}$ or symptomatic individuals delay seeking care. ${ }^{18}$ Post-deployment mental health screening may be an important link to both offset any lack in perceived need for mental healthcare and expediting care-seeking when it is needed.

In contrast, some authors have highlighted disadvantages to PDS and these generally relate to how a programme compares to the ideal. ${ }^{19}$ A successful PDS process is reliant on: (1) truthful reporting on screening tools that have good sensitivity and specificity for the conditions being measured, (2) appropriate follow through and adherence to recommended services, (3) effective treatment services for the identified concerns and (4) a beneficial opportunity cost for the investments in screening. However, for a given PDS programme each of these identified attributes may be present to greater or lesser degrees.

While there are potential benefits to PDS, a number of implementation characteristics can influence its utility. One such characteristic is the timing of screening. Some research has identified that there are disadvantages to screening too soon after a deployment. ${ }^{20-22}$ For instance, service members have been observed to report being non-symptomatic during their initial reintegration period post-deployment but then change to being symptomatic at a later time, ${ }^{20}$ perhaps due to delayed symptom development or members being less forthcoming with symptom reporting in the enthusiasm of returning home. Additionally, screening personnel have been observed to be more likely to recommend further follow-up care for service members without psychosocial concerns when screening shortly after a deployment ends, ${ }^{21}$ perhaps due to, correctly or incorrectly, assuming that some personnel were not fully forthcoming with symptom reporting. Moreover, service members may be reticent to screen shortly after a deployment as their perceptions of stigma and other barriers to care tends to be elevated for a period shortly after a deployment, ${ }^{22}$ which has the potential to impact symptom reporting. There are also disadvantages to screening too late as this undermines the primary goal of screening, identifying problems soon after deployment and reducing delays to care. While an optimal screening time has not been proposed, the Canadian Armed Forces $(\mathrm{CAF})$ requires that screening occur 90 to 180 days post-deployment. The US military and Australian military require two health assessments, an early screening and one that is to occur 90 to 180 days post-deployment. ${ }^{8}$

The CAF introduced its post-deployment mental health screening programme in 2002 and it became fully implemented in August of 2004. ${ }^{23}$ The fundamental goal of this screening has been to shorten the delays to care seen in those with deployment-related mental disorders, with a concomitant goal of screening for mental or physical health problems that may be unrelated to deployment. Additionally, PDS has been designed to provide feedback, guidance, education and advice on the post-deployment reintegration process and to reduce stigma surrounding mental illness. However, despite PDS in Canada and elsewhere having been in place for a number of years, limited research has explored service members' compliance. ${ }^{24}$ Such information would benefit attempts to improve the reach and effectiveness of these programmes and yet a comprehensive estimate of PDS compliance and associated factors has not been assessed in the CAF.

The present study addresses this research gap by using data from a cohort of Canadian military personnel, who deployed to various areas around the world, to assess service members' compliance with the 90 to 180 day PDS completion requirement and to investigate the factors associated with this compliance. Additionally, noting that, although lower, there is some utility in screening early ( $<90$ days) or late ( $>180$ days) relative to not screening at all, a secondary objective was to cursorily compare early, late and non-screeners. Moreover, a better understanding of screening compliance and its influences would help to identify targeted strategies that could potentially improve the adoption of screening across the full population at risk.

\section{METHODS}

\section{The post-deployment screening process}

The CAF has adjusted its mental health system over the past 15 years in an effort to minimise the impact of mental health problems on its members. ${ }^{25}$ Subtle changes were made in the application of medical policy that made it easier for personnel who recover from mental disorders to remain in uniform, reducing a potential career-related barrier to care seeking. The CAF also introduced a resilience and mental health training programme in 2008 with a focus on educating members on mental illness awareness and stigma reduction. This programme was further integrated across the deployment cycle in 2009 and included instruction with an additional emphasis on prevention and psychological resilience. In-depth post-deployment mental health screening was introduced in 2002 and it became fully implemented in the CAF in 2004.

Currently, CAF policy requires all service members who deploy to a special duty area for a period of 60 or more days to complete PDS between 90 and 180 days following deployment return; those deploying to some locations in Europe and the USA are exempted. Additionally, members who are already receiving mental healthcare are to also complete the process but without the time constraint. 
The PDS process begins with an orientation briefing of the screening's purpose, confidentiality and the associated follow-up. The member then completes a questionnaire that contains questions on sociodemographic and military characteristics, deployment experiences and health problems that are assessed using standardised instruments: the SF-36 Health Survey, a general health status measure with an emphasis on functioning ${ }^{26}$; portions of the Patient Health Questionnaire assessing physical symptoms, depression, suicidality, panic disorder and generalised anxiety disorder ${ }^{27}$; the Patient Checklist for PTSD-Civilian Version (PCL-C) ${ }^{28}$; the 10 -item Alcohol Use Disorder Identification Test ${ }^{29}$; a 30 item combat exposure scale ${ }^{30}$ and the Brief Traumatic Brain Injury Screen. ${ }^{31}$ These screening instruments were chosen to provide an indication of any concern in nine areas: (1) military-related post-traumatic stress disorder (PTSD) symptoms, (2) civilian-related PTSD symptoms, (3) depressive symptoms, (4) anxiety symptoms, (5) substance use, (6) post-concussive symptoms, (7) other physical health issues, (8) family/marital problems and (9) workplace conflict. The responses from these instruments are scored as indicated by the questionnaire guidelines and concerns are identified using either the recommended cut-offs or ones that were slightly adjusted for the CAF population. The responses and indicated concerns are then reviewed by a mental health professional who subsequently conducts a semi-structured interview. The interviewer then completes a 'tracking form' that captures a summary disposition of concerns about potential health problems in the above stated nine areas and an additional 'other' to be specified area, along with the interviewer's follow-up care recommendations. Approximately 6 to 8 weeks after the interview, the appointment register is reviewed to assess whether recommended follow-up care was received. Those without such follow-up are contacted to determine whether further care is still needed.

\section{Study population and sampling}

The study cohort consisted of all CAF personnel $(\mathrm{n}=28460)$ who had a deployment within the 01 January 2009 to 31 December 2014 time frame. This time frame was chosen partly based on the availability of electronic health records data to assist with sampling and also to ensure greater than 2 years of post-deployment follow-up among those not releasing from military service beforehand. A stratified random sample of 3004 individuals was identified from this cohort. Seven sampling strata were defined by administrative data that indicated increasing amounts of post-deployment mental health services use (MHSU) in order to guide over-sampling of those who would likely have a mental disorder: six strata categorised any speciality MHSU ( 0,1 to 3, $\geq 4$ psychiatrist or psychologist appointments) among those with and those without an indicated PDS-associated appointment and a seventh stratum included those with no identified MHSU in the administrative data. The study was powered to discern a delay to care difference of at least 50 days between screened and non-screened individuals with $85 \%$ power when employing a log-rank test. Sample size per stratum was determined using a Neyman optimal allocation approach. ${ }^{32}$ Medical records for 2997 individuals in the sample were reviewed and seven were inaccessible; 2598 individuals had a total of 2875 deployments that required PDS.

\section{Data collection}

Data on deployments were extracted from deployment tasking (extract date: 30 March 2016), deployment-related pay (extract date: 30 March 2016) and human resources (extract date: 01 August 2017) administrative databases. Clinical and other associated data were abstracted from paper and electronic medical records over the period of 06 February 2017 to 01 May 2018. PDS associated data was obtained from the medical records review and this was supplemented with electronic data from the PDS programme (extract date: 01 August 2012). Additional data on sociodemographic and military characteristics were obtained from human resources administrative data.

\section{Outcome measures}

The primary outcome assessed was compliant completion of PDS as indicated in the CAF PDS policy. Each deployment that required PDS was considered compliant only if the process was completed within a 90 to 180 days post-deployment time frame. Administrative data provided details on the sampled individuals' deployments over the period of interest (01 January 2009 to 31 December 2014).

A completed PDS occurred only when both the questionnaire and subsequent interview with a mental health professional were completed and this was based on documentation in either the medical record or electronic PDS programme data. The interview date determined the date of PDS completion. Those who screened early ( $<90$ days), late (>180 days) or in compliance (90 to 180 days), relative to the date of deployment return, were identified. Non-screeners were determined by the absence of documentation to indicate a completed screening (ie, a questionnaire and interview) in both the medical chart and electronic PDS programme data.

\section{Covariates of interest}

Previous research has identified a number of covariates to be associated with post-deployment mental health problems or delays to accessing care and these were considered for possibly influencing compliance with the PDS completion requirement. ${ }^{133}$ Arguably, individuals with these characteristics are the ones who would benefit most from PDS and as such, knowing whether these covariates are associated with compliance would be beneficial. First official language is among the sociodemographic covariates being considered; it has been found to be associated with better mental health statistics in some previous work ${ }^{4}$ and it may be a proxy for a base cluster as these service members tend to be concentrated in a subset of bases. The covariates of interest included: 
1. Military and sociodemographic information: age (19 to 24, 25 to 34,35 to 44 or 45 to 60 years), sex, ${ }^{34}$ first official language (English or French), ${ }^{4}$ marital status (married/commonlaw, divorced/separated/widowed or single - never married $),{ }^{35}$ years of military service $(<5$, 5 to 9,10 to 19 or $\geq 20$ years), rank category (junior non-commissioned member (JNCM), senior non-commissioned member (SNCM) or officer), ${ }^{1}$ component (Regular or Reserve Force), ${ }^{36}$ service (Army, Air Force or Navy) ${ }^{37}$ and combat arms military trade/ occupation. ${ }^{38}$

2. Deployment-related information: deployment location (Afghanistan or 'other'), deployment return date (2009 or $\geq 2010)$ and deployment length ( $\leq 180$ days, $>180$ days).

3. Mental health-related information: a past mental health problem documented in the medical chart and early MHSU interaction. In those screening early or compliant, an early MHSU interaction was defined as either a mental health diagnostic assessment or a MHSU appointment having been made on or before PDS; however, among those screening late or not at all, this early MHSU interaction would need to have occurred $\leq 180$ days post-deployment (ie, before the upper limit for compliant screening). The creation of an early MHSU interaction covariate was an attempt to identify and control for service members who receive mental healthcare soon after deployment return as these individuals are to also complete PDS but without the 90 to 180 day post-deployment constraint.

All time-varying covariates were assessed relative to deployment return date with the exception of marital status which was assessed on the human resources administrative data extract date. Variable categorisations were based on the data's distribution and previous work with this population.

\section{Statistical analysis}

The data were predominantly analysed using SAS for Windows, V.9.3 (SAS Institute Inc, North Carolina); marginal effect estimates were obtained using Stata for Windows, release 14 (StataCorp LP, College Station, Texas). We applied the sample design weights to determine descriptive and regression statistics and Taylor Series Linearisation ${ }^{39}$ was used to generate the associated SE estimates and 95\% CIs. The primary unit of analysis was deployment. Since some individuals (ie, 266 of 2598 sampled individuals) had multiple deployments, individuals were handled as cluster variables in the design specification to allow SEs to be corrected for the repeated measure. $^{40}$

List-wise deletion was used for missing values and this excluded less than $1 \%$ of the deployments; the deployment location covariate had a single missing value and all other covariates in the analyses were complete.

Descriptive statistics were generated and Wald $\mathrm{X}^{2}$ tests assessed associations between each variable and PDS compliance. While compliance with the 90 to 180 day post-deployment completion time was the main outcome, late completers ( $>180$ days), early completers ( $<90$ days) and non-completers were examined descriptively. KaplanMeier curves were generated to assess the time-to-PDS completion overall and by covariates that were determined to be significant in regression modelling; zero time was deployment return date, event date was PDS completion date and non-completers were censored at the start date of a subsequent deployment, their military release date or chart review date, whichever was earlier. Follow-up time for censored individuals ranged from a 72 day minimum to a 3128 day maximum (mean: 1640 days; median: 1634 days; IQR: 2214-1052=1162 days). Follow-up time for PDS completers ranged from 1 day to a maximum of 2345 days (mean: 167 days; median: 142 days; IQR: 114-183=69 days).

Logistic regression modelling investigated the association of covariates with PDS compliance, completion 90 to 180 days post-deployment. A conservative variable reduction strategy was used; variables were sequentially dropped if their covariate-adjusted Wald $\mathrm{X}^{2} \mathrm{p}$ value was $\geq 0.25$, starting with the variable having the largest $\mathrm{p}$ value.

We used a marginal standardisation approach to estimate the proportion of PDS-requiring deployments that, based on the final logistic regression model, would be expected to be compliant with screening had they had the characteristic of interest; this approach operates by statistically forcing the total population to have the characteristic of interest while other covariates retain their observed value. ${ }^{32}$ Expected marginal PDS compliance prevalence differences (MPD) were computed and a z-test assessed the MPD statistical significance with standard errors computed using the delta method. ${ }^{32}$

\section{Patient and public involvement}

CAF service members, patients and/or the public were not involved in developing the research question, the study design or in the conduct of the study. The findings from this study and the larger research project will be shared with CAF service members and other interested stakeholders through targeted conference venues, CAF community newsletters or communiques and other venues.

\section{RESULTS}

\section{Study population characteristics}

Table 1 summarises the demographic and military characteristics among unique individuals with deployments that required PDS during the study period (ie, 2009 to 2014). Individuals were predominantly English speaking, married, male, Regular Force members, in the JNCM rank category and Army service. On their first deployment return during the study period, a majority were less than 35 years of age (mean: 34.1 years, $95 \%$ CI 33.6 to 34.6) and just over half had been in military service for less than 10 years. Additionally, during the period of study 
Table 1 Military and sociodemographic characteristics for unique individuals with a deployment that required an postdeployment screening (sample $n=2598$, weighted $n=24664$ )

\section{Age* $^{*}$}

Characteristic

$19-24$

25-34 1040

$35-44 \quad 864$

45-60 398

Sex

Female 323

Male 2275

First official language

English 1810

French

788

Marital status $\dagger$

Married/common law 1775

Divorces/separated/widowed 258

Single $\quad 565$

Rank category*

$\begin{array}{lr}\text { JNCM } & 1552 \\ \text { SNCM } & 572 \\ \text { Officer } & 474\end{array}$

Years of military service ${ }^{\star}$

$\begin{array}{ll}<5 \text { years } & 449 \\ 5 \text { to } 9 \text { years } & 770 \\ 10 \text { to } 19 \text { years } & 827 \\ \geq 20 \text { years } & 552\end{array}$

$\geq 20$ years

Regular Force
Reserve Force
Service

\begin{tabular}{|c|c|c|}
\hline Army & 1792 & 70.3 (67.7 to 73.0$)$ \\
\hline Air Force & 505 & 17.0 (14.9 to 19.2$)$ \\
\hline Navy & 301 & 12.6 (10.7 to 14.6$)$ \\
\hline \multicolumn{3}{|l|}{ Combat arms occupation* } \\
\hline No & 1888 & 68.8 (66.1 to 71.6$)$ \\
\hline Yes & 710 & 31.2 (28.4 to 33.9 ) \\
\hline \multicolumn{3}{|l|}{ Deployment location } \\
\hline Other & 373 & 16.5 (14.3 to 18.6$)$ \\
\hline Afghanistan & 2225 & 83.5 (81.4 to 85.7$)$ \\
\hline \multicolumn{3}{|l|}{ Deployment locations } \\
\hline Afghanistan only & 2137 & 80.3 (78.0 to 82.7$)$ \\
\hline Other single location only & 356 & 15.4 (13.3 to 17.4$)$ \\
\hline Multiple locations $\ddagger$ & 105 & 4.3 (3.1 to 5.5$)$ \\
\hline \multicolumn{3}{|l|}{ Number of deployments } \\
\hline 1 & 2333 & 89.1 (87.3 to 91.0 ) \\
\hline 2 & 253 & 10.3 (8.6 to 12.2 ) \\
\hline
\end{tabular}

Continued
Weighted \% $(95 \% \mathrm{Cl})$

17.6 (15.3 to 19.9$)$

40.5 (37.6 to 43.4 )

27.3 (24.8 to 29.9$)$

14.6 (12.5 to 16.6$)$

10.2 (8.5 to 12.0$)$

89.8 (88.0 to 91.5$)$

70.1 (67.5 to 72.8 )

$29.9(27.2$ to 32.5$)$

66.8 (64.1 to 69.5$)$

6.4 (5.1 to 7.7 )

$26.8(24.2$ to 29.4$)$

58.4 (55.6 to 61.3$)$

19.6 (17.3 to 21.8$)$

22.0 (19.5 to 24.5$)$

24.0 (21.7 to 26.3$)$

29.1 (26.5 to 31.7$)$

25.8 (23.3 to 28.3$)$

21.1 (18.7 to 23.4 )

87.0 (85.1 to 89.0$)$

13.0 (11.0 to 14.9$)$

$19413.0(11.0$ to 14.9$)$

$0.3(67.7$ to 73.0$)$

17.0 (14.9 to 19.2$)$

12.6 (10.7 to 14.6$)$

68.8 (66.1 to 71.6 )

31.2 (28.4 to 33.9 )

16.5 (14.3 to 18.6)

83.5 (81.4 to 85.7 )

80.3 (78.0 to 82.7 )

15.4 (13.3 to 17.4 ) 
Table 1 Continued

\begin{tabular}{lcc}
\hline Characteristic & Sample $\mathbf{n}$ & Weighted \% (95\% Cl) \\
\hline 3 & 12 & 0.6 (0.1 to 1.0$)$ \\
\hline $\begin{array}{l}\text { Cumulative duration of deployments } \\
\quad<180 \text { days }\end{array}$ & 649 & $24.2(21.7$ to 26.6$)$ \\
$\quad 180-365$ days & 1791 & 69.4 (66.8 to 72.1$)$ \\
$\quad>365$ days & 158 & 6.4 (5.0 to 7.8$)$ \\
\hline Past mental health problem & & \\
$\quad$ No & 2413 & $97.6(97.0$ to 98.2$)$ \\
Yes & 185 & $2.4(1.8$ to 3.0$)$ \\
\hline
\end{tabular}

*The value for this covariate was calculated relative to individuals' first deployment return date during the study period.

†The value for this covariate was calculated at data extract date (extract date: 01 August 2017).

$\ddagger$ The multiple locations category could include either multiple 'other' location deployments or a combination of Afghanistan and 'other' location deployments.

JNCM, junior non-commissioned member; SNCM, senior non-commissioned member.

$89.1 \%$ of individuals had only one deployment and $83.5 \%$ had an Afghanistan deployment.

\section{Post-deployment screening completion and compliance}

Figure 1 characterises the cumulative proportion of deployments with a PDS requirement that had one completed as post-deployment time increases (ie, KaplanMeier estimates); while the rate of completions tended to decrease after 180 days, the completions did not plateau until 390 to 450 days post-deployment. An estimated $67.3 \%$ (95\% CI 65.0 to 69.6 ) of deployments that required a PDS had one completed; $43.3 \%$ (95\% CI 40.6 to 46.0 ) were completed within the 90 to 180 day post-deployment period (policy compliant), $6.5 \%$ (95\% CI 5.0 to 7.9) were completed early (ie $<90$ days post-deployment) and $17.6 \%$ (95\% CI 15.4 to 19.7 ) were completed late

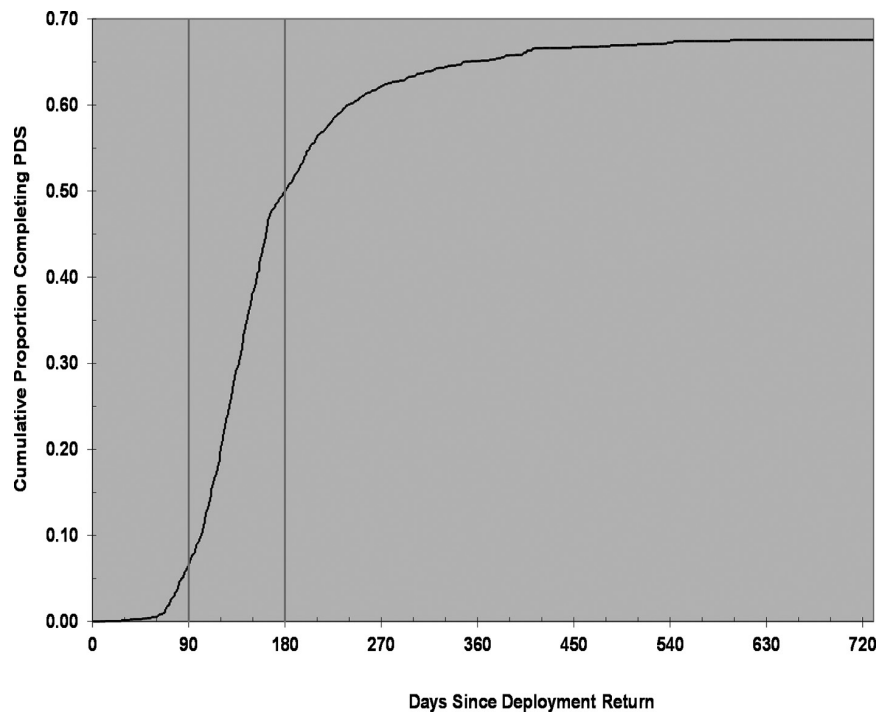

Figure 1 Cumulative proportion of deployments occurring over the 2009 to 2014 period that required post-deployment screening and had one completed as post-deployment time increased. The compliant completion period was 90 to 180 days post-deployment. PDS, post-deployment screening. (ie, $>180$ days post-deployment). Moreover, contrary to expectations, $16.8 \%$ of deployments that did not require PDS had one completed; these deployments and associated PDS were not assessed further.

Service members who receive mental healthcare soon after deployment return are to also complete PDS but without the 90 to 180 day post-deployment constraint. We identified individuals who had interactions with mental health services post-deployment but prior to screening or, for non-screeners and late completers, prior to the upper time cut-off for screening compliance (ie, 180 days post-deployment). In many instances, such individuals are compliant screeners as they do not have to screen within a certain time period if they were receiving care before this 180 day post-deployment period. Overall, $3.3 \%$ (95\% CI 2.6 to 3.9) of deployments were from individuals with an early MHSU interaction. Breaking this down by PDS completion status, $2.6 \%$ of compliant completers, $0.6 \%$ of early completers, $4.0 \%$ of late completers and $4.2 \%$ of non-completers had an early MHSU interaction. If all non-completers with this early interaction ultimately complete a PDS, completed screenings would increase from $67.3 \%$ to $68.7 \%$ (95\% CI 66.4 to 71.0 ). Similarly, if all those who were identified as non-compliant completers but had this early MHSU interaction were designated compliant screeners, the compliant screening estimate would increase from $43.3 \%$ to $45.4 \%$ (95\% CI 42.7 to 48.1 ).

We further looked at MHSU interactions among both late completers and non-completers to identify the fraction that may have benefitted from earlier screening and $32.9 \%$ (95\% CI 28.0 to 37.9 ) of late completers had a MHSU interaction; this was comprised of $28.9 \%$ (95\% CI 24.0 to 33.8) with an interaction that was more than 180 days post-deployment and $4.0 \%$ that were earlier. In comparison, $20.5 \%$ (95\% CI 17.7 to 23.2 ) of non-completers had a MHSU interaction; this was comprised of $16.2 \%$ (95\% CI 13.7 to 18.8 ) with an interaction that 
was more than 180 days post-deployment and $4.2 \%$ that were earlier. Thus, $28.9 \%$ of late completers and $16.2 \%$ of non-completers might have had a shortened delay to care had they been compliant screeners.

The sociodemographic and military characteristics of individuals with deployments that required PDS are provided in table 2 by PDS completion status. The three non-compliant completion groups (ie, not completed, early completion and late completion) differed on most covariates. Notably, those not completing PDS were more likely to be older, in higher ranks, in non-combat arms occupations, Navy (less from the Army), have a non-Afghanistan deployment location and have shorter deployments (ie, $\leq 180$ days) relative to both early and late completers. Other univariate differences were such that non-completers were more likely to have more years of military service, a more recent deployment return date (ie, 22010) and to be Regular Force members relative to late completers. Additionally, relative to early completers, non-completers were more likely to have an English first official language, a less recent deployment return date and an early MHSU interaction.

Additionally, Wald $\mathrm{X}^{2}$ tests comparing covariates among the early, late and compliant completion groups indicated that they were largely similar. However, relative to the other two groups early completers were more likely to have a first official language that was French and a more recent deployment return date (ie, $\geq 2010$ ), but less likely to have an early MHSU interaction. Late completers were more likely to have shorter deployments (ie, $\leq 180$ days).

Wald $\mathrm{X}^{2}$ tests comparing the compliant and non-compliant (overall) completion groups indicated that the non-compliant group was more likely to be older, in higher ranks, have more years in military service, Navy (less from the Army), in non-combat arms occupations, have a non-Afghanistan deployment location and have shorter deployments relative to the compliant group (table 2).

\section{Logistic regression results}

Table 3 presents the logistic regression analysis for PDS completion compliance. Five covariates were excluded in the final variable-reduced model: years of military service, deployment return date, service, age category and marital status. We observed that the odds of PDS completion compliance was significantly associated with a number of covariates. Compliance was higher for the JNCM rank category relative to officers (adjusted OR (AOR): 1.59; 95\% CI 1.17 to 2.16), combat arms occupations (AOR: 1.44; $95 \%$ CI 1.11 to 1.86 ) and Afghanistan deployments (AOR: 2.37; 95\% CI 1.62 to 3.47); it was lower for shorter deployments (ie, $\leq 180$ days relative to $>180$ days) (AOR: 0.65 ; $95 \%$ CI 0.48 to 0.86 ) and among personnel with a past mental health problem (AOR: $0.65 ; 95 \%$ CI 0.42 to $1.00)$. Completion compliance was lower with marginal significance $(0.05<\mathrm{p} \leq 0.10)$ among those whose first official language was French (AOR: 0.79; 95\% CI 0.61 to 1.03 ) and those with an early MHSU interaction (AOR: 0.63;
$95 \%$ CI 0.39 to 1.05$)$. Completion compliance differences were not significant for the sex and component covariates, or for SNCM ranks relative to officers.

Marginal PDS compliance prevalence difference estimates are presented in table 3. PDS completion compliance was $10.6 \%$ higher for JNCM ranks relative to officers, 8.4\% higher for the combat arms occupation, 19.2\% higher for Afghanistan deployments, 10.1\% higher for longer deployments (>180 days) and 9.7\% higher in those without a past mental health problem.

Figure 2 characterises the cumulative proportion of deployments with a PDS requirement that had one completed as post-deployment time increases (ie, KaplanMeier estimates) for statistically significant covariates identified by the logistic regression analysis. There are three time intervals of interest. The rate of early PDS completion appears to be higher for Afghanistan deployments and deployments greater than 180 days, relative to their counterpart covariate values. Differences for the compliant PDS completion period are reflective of what was noted in the logistic regression analysis. However, for Afghanistan deployment locations, combat arms occupations and non-commissioned member (NCM) ranks (both JNCM and SNCM) the rate of late PDS completions appears to continue at an elevated level relative to their counterpart covariate value. In contrast, the late completion rate appears to increase comparably for both deployment length covariate values (ie, $\leq 180$ days, $>180$ days) before the cumulative proportion plateaus. The rate of late PDS completions for those with a past mental health problem appears to be more variable; initially elevated then comparable or lower relative to those without a past mental health problem. Moreover, it was observed that among individuals with each of these more-likely-toscreen characteristics, the cumulative proportion with a completed PDS approached $80 \%$ or less as time passed.

\section{DISCUSSION}

\section{Key findings}

The objective of the current study was to estimate PDS completion compliance, as measured by completion of screening 90 to 180 days post-deployment for deployments that required it, and to examine factors associated with this compliance. We found that $67.3 \%$ of deployments that required PDS had one completed and $43.3 \%$ were completed within the compliant post-deployment period. PDS compliance was higher with a lower rank category (10.6\% for JNCM relative to officers), combat arms occupations (8.4\%), Afghanistan deployments (19.2\%), longer deployments $(10.1 \%)$ and among those without a past mental health problem $(9.7 \%)$. Additionally, screening compliance was higher with marginal significance among those whose first official language was English and those without an early MHSU interaction.

The rate of late PDS completions was observed to continue at an elevated level as time passed beyond the upper time limit for compliance (ie, >180 days 


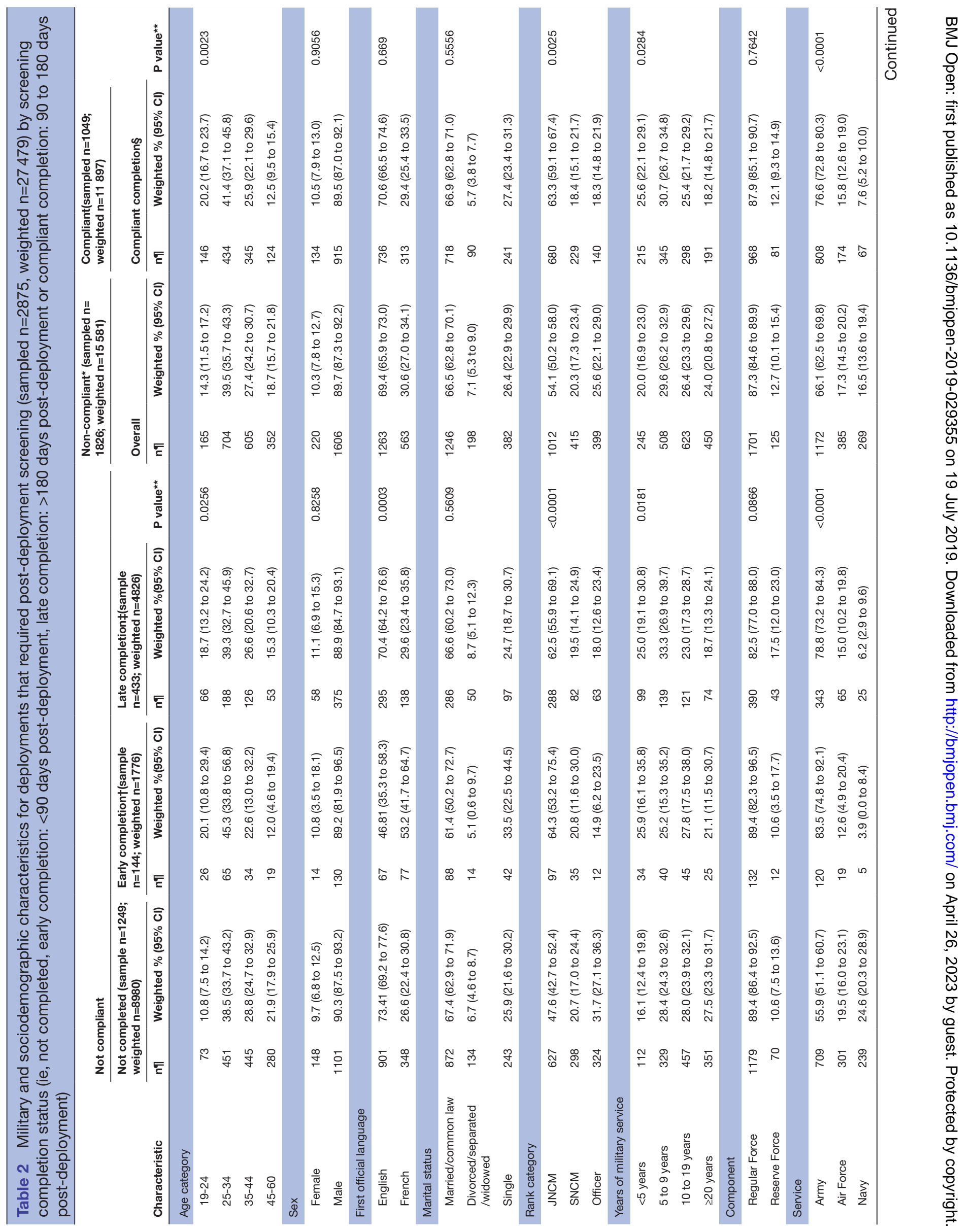




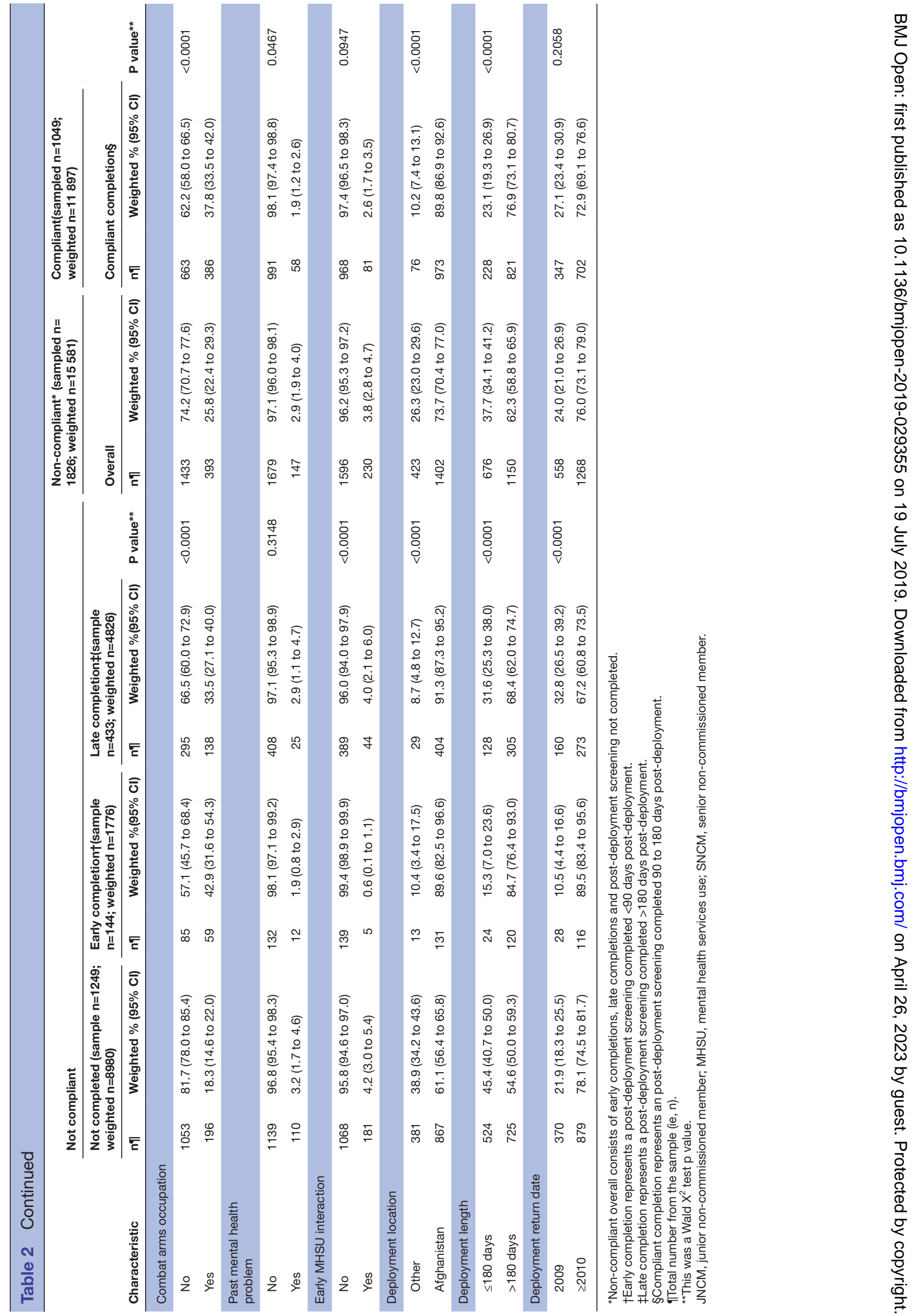


Table 3 Logistic regression results for associations with post-deployment screening compliance, completion 90 to 180 days post-deployment and marginal prevalence estimates for screening compliance among Canadian military personnel

\begin{tabular}{|c|c|c|c|c|}
\hline \multirow[b]{2}{*}{ Characteristic* $^{*}$} & \multirow[b]{2}{*}{ AOR $(95 \% \mathrm{Cl})$} & \multicolumn{3}{|c|}{ Marginal PDS compliance prevalence $(\%(95 \% \mathrm{CI}))$} \\
\hline & & Estimate & $\begin{array}{l}\text { Difference (estimate } \\
\text { minus reference value) }\end{array}$ & P value† \\
\hline \multicolumn{5}{|c|}{ First official language } \\
\hline French & $0.79 \ddagger(0.61$ to 1.03$)$ & 39.7 (34.7 to 44.6$)$ & $-5.3 \ddagger(-11.3$ to 0.6$)$ & 0.0693 \\
\hline English & Reference & $45.0(41.8$ to 48.1$)$ & Reference & \\
\hline \multicolumn{5}{|l|}{ Sex } \\
\hline Female & 1.34 (0.92 to 1.96$)$ & 49.5 (41.2 to 57.7$)$ & $6.8(-1.9$ to 15.5$)$ & 0.1343 \\
\hline Male & Reference & 42.7 (39.9 to 45.4$)$ & Reference & \\
\hline \multicolumn{5}{|l|}{ Rank category } \\
\hline JNCM & $1.59 \S(1.17$ to 2.16$)$ & 46.7 (43.2 to 50.3$)$ & $10.6 \S(3.8$ to 17.4$)$ & 0.0125 \\
\hline SNCM & $1.26(0.86$ to 1.84$)$ & 41.3 (35.3 to 47.4$)$ & $5.2(-3.3$ to 13.7$)$ & 0.2651 \\
\hline Officer & Reference & 36.2 (30.4 to 41.9$)$ & Reference & \\
\hline \multicolumn{5}{|l|}{ Component } \\
\hline Reserve Force & 0.77 (0.53 to 1.11$)$ & 38.1 (30.5 to 45.8$)$ & $-6.0(-14.1$ to 2.1$)$ & 0.1414 \\
\hline Regular Force & Reference & 44.1 (41.3 to 46.9$)$ & Reference & \\
\hline \multicolumn{5}{|c|}{ Combat arms occupation } \\
\hline Yes & $1.44 \S(1.11$ to 1.86$)$ & 49.1 (44.1 to 54.0$)$ & $8.4 \S(2.4$ to 14.5$)$ & 0.0107 \\
\hline No & Reference & 40.6 (37.4 to 43.9$)$ & Reference & \\
\hline \multicolumn{5}{|c|}{ Past mental health problem } \\
\hline Yes & $0.65 \S(0.42$ to 1.00$)$ & 33.9 (25.0 to 42.9$)$ & $-9.7 \S(-19.0$ to -0.4$)$ & 0.0378 \\
\hline No & Reference & 43.6 (40.9 to 46.3$)$ & Reference & \\
\hline \multicolumn{5}{|c|}{ Early MHSU interaction } \\
\hline Yes & $0.63 \ddagger(0.39$ to 1.05$)$ & 33.6 (23.4 to 43.8$)$ & $-10.1 \ddagger(-20.6$ to 0.4$)$ & 0.0571 \\
\hline No & Reference & 43.7 (41.0 to 46.4$)$ & Reference & \\
\hline \multicolumn{5}{|c|}{ Deployment location } \\
\hline Afghanistan & $2.37 \S(1.62$ to 3.47$)$ & 46.8 (43.8 to 49.9$)$ & $19.2 \S(11.5$ to 27.0$)$ & 0.0021 \\
\hline Other & Reference & 27.6 (20.8 to 34.4$)$ & Reference & \\
\hline \multicolumn{5}{|l|}{ Deployment length } \\
\hline$\leq 180$ days & $0.65 \S(0.48$ to 0.86$)$ & 36.2 (30.9 to 41.6$)$ & $-10.1 \S(-16.6$ to -3.6$)$ & 0.0012 \\
\hline$>180$ days & Reference & 46.3 (43.1 to 49.6$)$ & Reference & \\
\hline
\end{tabular}

${ }^{*}$ A variable-reduction strategy was used (ie, sequentially drop the covariate with the largest Wald $X^{2} p$ value, if $p>0.25$ ) and this resulted in five variables being dropped: (1) years of military service ( $p=0.9593),(2)$ deployment return date $(p=0.9217),(3)$ service $(p=0.6930),(4)$ age category $(p=0.4279)$ and (5) marital status $(p=0.3710)$.

†A z-test assessed the statistical significance of marginal screening compliance prevalence differences and associated SEs were computed using the delta method.

$\ddagger$ Significant at $0.05<\mathrm{p} \leq 0.10$.

$\S$ Significant at $p \leq 0.05$.

AOR, adjusted OR; JNCM, junior non-commissioned member; MHSU, mental health services use; PDS, post-deployment screening; SNCM, senior non-commissioned member.

post-deployment) among individuals with an Afghanistan deployment, a combat arms occupation and NCM ranks (both JNCM and SNCM) relative to their counterpart covariate values. This may reflect a delayed recognition of need for care, with PDS used as a means to achieve this, or it may simply be reflective of a late attempt to be compliant with PDS. However, we also observed that $28.9 \%$ of late completers and, additionally, $16.2 \%$ of non-completers had a MHSU interaction more than 180 days post-deployment. This suggests a delay to care in a sizeable fraction of late and non-screeners that may have possibly been averted had they screened sooner, especially if the MHSU was related to a prior deployment.

\section{Comparison with other research}

We were unable to locate much research on compliance with PDS in military personnel from other countries. In the US military, PDS consists of a post-deployment health 

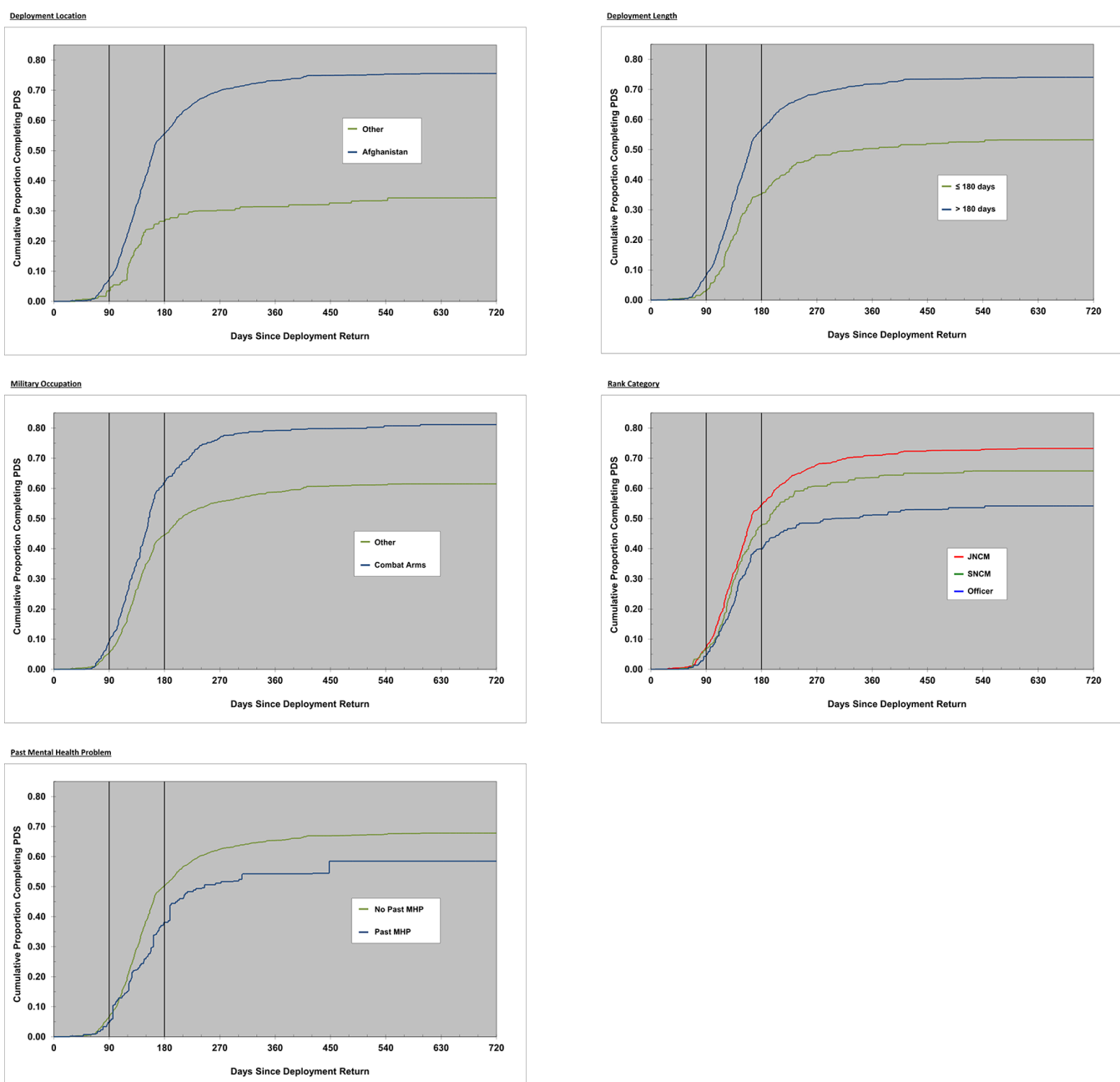

Figure 2 Cumulative proportion of deployments occurring over the 2009 to 2014 period that required PDS and had one completed as post-deployment time increased by specific covariates. All covariates had a statistically significant association with compliant PDS completion (ie, 90 to 180 days post-deployment) as determined by multiple logistic regression. PDS, postdeployment screening.

assessment shortly (ie, approximately 30 days) after a deployment ends and a second health status reassessment 90 to 180 days post-deployment; these assessments use standardised screening questionnaires and a meeting with a healthcare provider. ${ }^{24}$ Some research has estimated compliance with the initial post-deployment health assessment screening to be $76.8 \%$ and this reducing to $33.5 \%$ for the reassessment screening, estimated among 289355 US service members returning from deployments (ie, Operation Iraqi Freedom) ending in $2005 .{ }^{24}$ The researchers suggested that service members who were female, in the Army or Air Force, Reserve component and in non-combat occupations were more likely to complete a screening; however, this interpretation was based on univariate summary statistics without formal statistical testing. Our findings were a little different; $67.3 \%$ of required screenings were completed, and we did not observe statistically significant differences in compliant completions (ie, 90 to 180 days post-deployment) for sex, service or component covariates and we observed a reverse finding for combat arms occupations as they were $8.5 \%$ more compliant.

\section{Limitations}

The primary limitation of our study relates to it being retrospective. While we were able to identify records and dates for deployment information, PDS completion and other associated details, we were reliant on the information that was available. We were unable to identify specific individual reasons for non-compliance, particularly for those who didn't complete a required PDS. Some individuals would have completed their PDS early or late, or not yet, because they already had interactions with mental health services and a completed screening among such individuals is an administrative requirement without a time constraint; we estimate that total completions and compliant completions would have only increased by approximately $1.4 \%$ and $2.1 \%$, respectively, for this reason. Additionally, we determined PDS completion date based on the date of the summary 'tracking form', which is completed following the interview with a mental 
health professional at the end of screening. An unknown number of individuals may have started the screening process prior to the required 180 day post-deployment period but finished it after this time point, or started without continuing on to complete the interview; these individuals would have been classified as non-compliant late completers, or non-completers in those without an interview. Moreover, while screening in the CAF requires that personnel returning from requisite deployments complete PDS 90 to 180 days after deployment return, this study and assessment was not designed to determine whether this screening window is optimal with respect to desired outcomes for the CAF setting.

\section{Interpretation}

In the CAF, the responsibility for mental health is shared among a member's command, clinicians and the member, with each having different but overlapping responsibilities that vary across the deployment cycle. Nevertheless, compliance with PDS is largely the responsibility of the member's current commanding officer. We found that the compliance with the PDS requirement, as measured by completion timing, was less than ideal for deployments that required individuals to undergo screening. Both the fraction of individuals who completed a screening $(67 \%)$ and those who completed it within the compliance time frame (43\%) were lower than expected and this suggests that there is a need for improvement. The CAF does not currently have a formal process to report PDS completion statistics for the review of commanding officers. Our findings suggest that the implementation of such feedback mechanisms would be beneficial, especially if the lag from deployment return to compliance reporting was optimised. This would assist in clarifying where the barriers to compliance may occur (ie, at the service member or commanding officer level) and it would allow for adjustments that could potentially enhance compliance.

In order to better understand the influences on PDS completion compliance, we investigated a number of factors that had the potential to influence screening compliance and this indicated that selection processes may be occurring with respect to the characteristics of individuals who were more likely to screen. A number of factors that have previously been identified as having some association with developing post-deployment mental health problems, and are commonly known among military personnel, were observed to also have a strong influence on compliance with screening. Specifically, deployments to Afghanistan, longer deployments, lower ranks and combat occupations have each been found to be associated with a higher rate of post-deployment mental health problems ${ }^{12414}$ and these characteristics were associated with higher compliance. However, even among individuals with these characteristics PDS completion only approaches $80 \%$ when the compliance time constraint is ignored, and this would be closer to approximately $50 \%$ when imposing the compliance time constraint. Therefore, while those who are more likely to have post-deployment mental health problems are more likely to undergo PDS, moderately large fractions with these characteristics do not. Additionally, the lower completion compliance that was observed among those with a past mental health problem and officers suggests that some service members may be hesitant to screen, perhaps because of some perceived barrier. ${ }^{43}{ }^{44}$ Our findings would suggest that there is a need to reinforce the guidelines for PDS among both commanding officers and service members, perhaps as part of their mental health training, in order to optimise compliance with screening completion. This would help to ensure that all members receive the same direction post-deployment, possibly reducing some individuals' hesitancy or avoidance. It may also help to reduce the number of individuals who screen too early or too late and possibly, erroneously, perceive their screening to be compliant.

In defining compliance with completion of PDS, we specified that non-compliance can occur when a screening is completed outside of the 90 to 180 day post-deployment period as well as when not completed at all. The rationale for a compliant screening period relates to implied disadvantages to either screening too early (eg, returnee's reduced likelihood of reporting symptoms immediately post-deployment) ${ }^{20} 2224$ or screening too late, which undermines the goal of early problem detection. We observed some indication that a fraction of late screeners were seeking mental health services after the 180 day post-deployment period, possibly supporting the notion of a delay-tocare disadvantage to screening late. However, a more detailed assessment of the timing of PDS completion and its influence on outcomes such as delay to care and both screening sensitivity and specificity are needed to further delineate the optimal period of completion. Additionally, while PDS is designed to identify potential mental health problems that may require a follow-up mental health assessment and care, further work is also needed to assess what happens after this. For example, it would be beneficial to determine whether certain screening-associated or individual characteristics influence outcomes such as a follow-up recommendation, compliance with a follow-up recommendation and ultimately, MHSU and a mental disorder diagnosis.

\section{CONCLUSIONS}

Post-deployment screening in the CAF is required for all service members who return from certain international deployments. We found that $67.3 \%$ of deployments that required screening had one completed and $43.3 \%$ were completed within the compliant post-deployment period. Afghanistan deployments, longer deployments, lower ranks, combat occupations and absence of a past mental health problem were associated with greater compliant screening. Our findings suggest a need to reinforce feedback mechanisms on PDS completion for the review of commanding officers as well as further instruction on the 
guidelines and objectives of PDS for both commanding officers and service members in order to reduce PDS non-compliance.

Acknowledgements We thank Julie Lanouette for her assistance with the data collection from patient medical records. We also thank Dr Bryan Garber for his comments on the study results and its interpretation. Additionally, we thank Dr. Bryan Garber, Dr. Minh Do, Dr. Mark Zamorski and Dr. Corneliu Rusu for their participation in the initial design and implementation of the overarching study.

Contributors DB had full access to all data in the study and takes responsibility for the integrity of the data and accuracy of the data analysis. PJHB and DJ implemented the data cleaning for the study variables. PJHB, DB and DJ wrote portions of initial drafts of the manuscript. DB finalised the analysis and writing of the manuscript and was principal investigator of the study. PJHB, DB and DJ have read and agree with the manuscript's final content.

Funding This work was supported by funding from the Canadian Armed Forces Surgeon General's Medical Research Program.

Competing interests PJHB is a serving member of the Canadian Armed Forces. DB and DJ are employees of the Canadian Department of National Defence and funding for this research came via this federal government department.

Patient consent for publication Not required.

Ethics approval This research was approved by IRB Services, 372 Hollandview Trail, Suite 300, Aurora, Ontario, Canada.

Provenance and peer review Not commissioned; externally peer reviewed.

Open access This is an open access article distributed in accordance with the Creative Commons Attribution Non Commercial (CC BY-NC 4.0) license, which permits others to distribute, remix, adapt, build upon this work non-commercially, and license their derivative works on different terms, provided the original work is properly cited, appropriate credit is given, any changes made indicated, and the use is non-commercial. See: http://creativecommons.org/licenses/by-nc/4.0/.

\section{REFERENCES}

1. Boulos D, Zamorski MA. Deployment-related mental disorders among Canadian Forces personnel deployed in support of the mission in Afghanistan, 2001-2008. CMAJ 2013;185:E545-52.

2. Fear NT, Jones M, Murphy D, et al. What are the consequences of deployment to Iraq and Afghanistan on the mental health of the UK armed forces? A cohort study. Lancet 2010;375:1783-97.

3. Hosek J. How Is Deployment to Iraq and Afghanistan Affecting US Service Members and Their Families? An Overview of Early RAND Research on the Topic. RAND Corporation 2011.

4. Zamorski MA, Rusu C, Garber BG. Prevalence and correlates of mental health problems in Canadian Forces personnel who deployed in support of the mission in Afghanistan: findings from postdeployment screenings, 2009-2012. Can J Psychiatry 2014;59:319-26.

5. Adler AB, Wright KM, Huffman AH, et al. Deployment cycle effects on the psychological screening of soldiers.. US Army Medical Department Journal 2000;4/5/6:31-7.

6. Bliese P, Wright K, Adler A, et al. Post-deployment psychological screening: Interpreting and scoring DD Form 2900. US Army Medical Research Unit-Europe Research Report 2005:3.

7. Lee DJ, Warner CH, Hoge CW. Advances and controversies in military posttraumatic stress disorder screening. Curr Psychiatry Rep 2014;16:467.

8. O'Donnell M, Dell L, Fletcher S, et al. The Australian Defence Force Mental Health Screening Continuum Framework: Full Report. Canberra: Department of Defence, 2014.

9. Vermetten E, Greenberg N, Boeschoten MA, et al. Deploymentrelated mental health support: comparative analysis of NATO and allied ISAF partners. Eur J Psychotraumatol 2014;5:23732.

10. Buckman JE, Forbes HJ, Clayton T, et al. Early Service leavers: a study of the factors associated with premature separation from the UK Armed Forces and the mental health of those that leave early. Eur J Public Health 2013;23:410-5.

11. Maguen S, Madden E, Neylan TC, et al. Timing of mental health treatment and PTSD Symptom improvement among Iraq and Afghanistan veterans. Psychiatr Serv 2014;65:1414-9.

12. Boulos D, Zamorski MA. Do shorter delays to care and mental health system renewal translate into better occupational outcome after mental disorder diagnosis in a cohort of Canadian military personnel who returned from an Afghanistan deployment? BMJ Open 2015:5:e008591.

13. Tsiachristas A, Thomas T, Leal J, et al. Economic impact of early intervention in psychosis services: results from a longitudinal retrospective controlled study in England. BMJ Open 2016;6:e012611.

14. Fikretoglu D, Guay S, Pedlar D, et al. Twelve month use of mental health services in a nationally representative, active military sample. Med Care 2008;46:217-23.

15. Hines LA, Goodwin L, Jones $M$, et al. Factors affecting help seeking for mental health problems after deployment to Iraq and Afghanistan. Psychiatr Serv 2014:65:98-105.

16. Hoge CW, Castro CA, Messer SC, et al. Combat duty in Iraq and Afghanistan, mental health problems, and barriers to care. $N$ Engl $J$ Med 2004;351:13-22.

17. Fikretoglu D, Liu A, Zamorski MA, et al. Perceived need for and perceived sufficiency of mental health care in the canadian armed forces: changes in the past decade and comparisons to the general population. Can J Psychiatry 2016;61:36S-45.

18. Wang PS, Berglund P, Olfson M, et al. Failure and delay in initial treatment contact after first onset of mental disorders in the National Comorbidity Survey Replication. Arch Gen Psychiatry 2005;62:603-13.

19. Panaite V, Brown R, Henry M, et al. Post-deployment mental health screening: a systematic review of current evidence and future directions. Adm Policy Ment Health 2018;45:850-75

20. Bliese PD, Wright KM, Adler AB, et al. Timing of postcombat mental health assessments. Psychol Serv 2007;4:141-8.

21. Zamorski MA. Timing of Enhanced Post-Deployment Screening: Exploration of Participants' Preferences and of the Associations among Timing, the Prevalence of Health Problems, and the Likelihood of Referral. In: Human Dimensions in Military Operations Military Leaders' Strategies for Addressing Stress and Psychological Support (pp. 32-1 - 32-22). Meeting Proceedings RTO-MPHFM-134, Paper 32. Neuilly-sur-Seine. France: RTO, 2006.

22. Osório C, Jones N, Fertout M, et al. Perceptions of stigma and barriers to care among UK military personnel deployed to Afghanistan and Iraq. Anxiety Stress Coping 2013;26:539-57.

23. Bailey S. The Canadian Forces Health Services Road to Mental Readiness Programme. Medical Corps Int Forum 2015:37-48.

24. Martin C. Routine screening and referrals for Post-Traumatic Stress Disorder after returning form Operation Iraqi Freedom in 2005. US Armed Forces MSMR 2007;14:2-7.

25. The Department of National Defence and The Canadian Armed Forces. Surgeon General's Mental Health Strategy: Canadian Forces Health Services Group - An Evolution of Excellence. https://www. canada.ca/content/dam/dnd-mdn/migration/assets/FORCES Internet/docs/en/about-reports-pubs-health/surg-gen-mental-healthstrategy.pdf (Accessed 03 Apr 2019).

26. Ware JE, Kosinski M. SF-36 physical \& mental health summary scales: a manual for users of version 1: Quality Metric. 2001.

27. Spitzer RL, Kroenke K, Williams JB. Group PHQPCS. Validation and utility of a self-report version of PRIME-MD: the PHQ primary care study. Jama 1999;282:1737-44.

28. Weathers FW, Litz BT, Herman DS, et al. The PTSD Checklist (PCL): reliability, validity, and diagnostic utility. annual convention of the international society for traumatic stress studies. San Antonio, TX, 1993.

29. Babor TF, de la Fuente JR, Saunders J, et al. The Alcohol Use Disorders Identification Test: Guidelines for use in. 2011

30. Office of the Surgeon General U. Mental Health Advisory Team (MHAT) IV: Operation Iraqi Freedom 05-07. Washington, DC: US Army Medical Command, 2006.

31. Schwab KA, Ivins B, Cramer G, et al. Screening for traumatic brain injury in troops returning from deployment in Afghanistan and Iraq: initial investigation of the usefulness of a short screening tool for traumatic brain injury. J Head Trauma Rehabil 2007;22:377-89.

32. Cochran WG. Sampling techniques: John Wiley \& Sons, 2007.

33. Boulos D, Zamorski MA. Delay to mental healthcare in a cohort of Canadian Armed Forces personnel with deployment-related mental disorders, 2002-2011: a retrospective cohort study. BMJ Open 2016;6:e012384.

34. Woodhead C, Wessely S, Jones N, et al. Impact of exposure to combat during deployment to Iraq and Afghanistan on mental health by gender. Psychol Med 2012;42:1985-96.

35. Browne T, Hull L, Horn O, et al. Explanations for the increase in mental health problems in UK reserve forces who have served in Iraq. Br J Psychiatry 2007;190:484-9.

36. Griffith J. Decades of transition for the US reserves: changing demands on reserve identity and mental well-being. Int Rev Psychiatry 2011;23:181-91. 
37. Center AFHS. Update: deployment health assessments, US Armed Forces, February 2010. MSMR 2010;17:18-19.

38. Weber E. Deployment Limiting Mental Health Conditions in US Military Personnel Deployed to Combat Theaters: Predictors of Theater Mental Health Evacuation. Journal of Psychology \& Clinical Psychiatry 2015;2.

39. Wolter K. Introduction to variance estimation: Springer Science \& Business Media. 2007.

40. Agnelli R. Examples of Logistic Modeling with the SURVEYLOGISTIC Procedure. In: Proceedings of the SAS Global Forum 2014 Conference, SAS Global Forum, Washington, DC, Mar 23-26, 2014. Cary, NC: SAS Institute Inc, 2014

41. Smith TC, Ryan MA, Wingard DL, et al. New onset and persistent symptoms of post-traumatic stress disorder self reported after deployment and combat exposures: prospective population based US military cohort study. BMJ 2008;336:366-71.

42. Boulos D, Fikretoglu D. Influence of military component and deployment-related experiences on mental disorders among Canadian military personnel who deployed to Afghanistan: a crosssectional survey. BMJ Open 2018;8:e018735.

43. Keeling M, Knight T, Sharp D, et al. Contrasting beliefs about screening for mental disorders among UK military personnel returning from deployment to Afghanistan. $J$ Med Screen 2012;19:206-11.

44. Rona RJ, Burdett $\mathrm{H}$, Khondoker M, et al. Post-deployment screening for mental disorders and tailored advice about help-seeking in the UK military: a cluster randomised controlled trial. Lancet 2017;389:1410-23. 